\title{
Unusual self revealing nuclear segmentation in a neutrophil from primary bone marrow cells in Mus musculus
}

\author{
Anthony Swain $\cdot$ D. Rhodri E. Jones
}

Received: 3 September 2014 / Accepted: 4 September 2014 /Published online: 23 September 2014

(C) Springer-Verlag London 2014

\begin{abstract}
This article describes unique species-specific neutrophil morphology in mouse bone marrow and suggests a possible correlation.
\end{abstract}

Keywords Mus musculus $\cdot$ Neutrophil segmentation

During the course of screening experiments, a 1-year-old ICR mouse was sacrificed and a bone marrow sample obtained by splitting a femur. The mononuclear cell layer was harvested and cultured for 5 days in minimum essential medium (MEM; Wako) in the presence of the following cytokines: stem cell factor (SCF $5 \mu \mathrm{l} / \mathrm{ml}$, PeproTech), interleukin-3 (IL-3 $5 \mu \mathrm{l} / \mathrm{ml}$, PeproTech), and erythropoietin (EPO $1 \mu \mathrm{l} / \mathrm{ml}$, R\&D Systems) with an addition of penicillin/streptomycin $(1 \mu \mathrm{l} / \mathrm{ml}$, Sigma) and $15 \% v / v$ heat-treated fetal bovine serum (FBS; Life Technologies). Cells were centrifuged (Shandon Cytospin $3^{\circledR}$ ), and morphology was observed under light microscopy after staining with May Grunwald/Giemsa. After scanning a number of fields, one neutrophil was observed with uncommon nuclear segmentation, perhaps giving an indication of the origin of this particular sample (Fig. 1). Although unusual, such findings are not without precedent. Unusual neutrophil nuclear morphology has recently been reported in a human patient (Bitar and Saba 2014), where accurate enumeration of the cells with distinct nuclei could be achieved.

We look forward to more reports of unusual nuclear morphology, perhaps in exotic species.

A. Swain $(\bowtie) \cdot$ D. R. E. Jones

Department of Research and Development of Next Generation Medicine, Faculty of Medical Sciences, Biomedical Research Station 211, Kyushu University, 3-1-1 Maidashi, Higashi-Ku, Fukuoka 812-8582, Japan

e-mail: anthony@jsd.med.kyushu-u.ac.jp

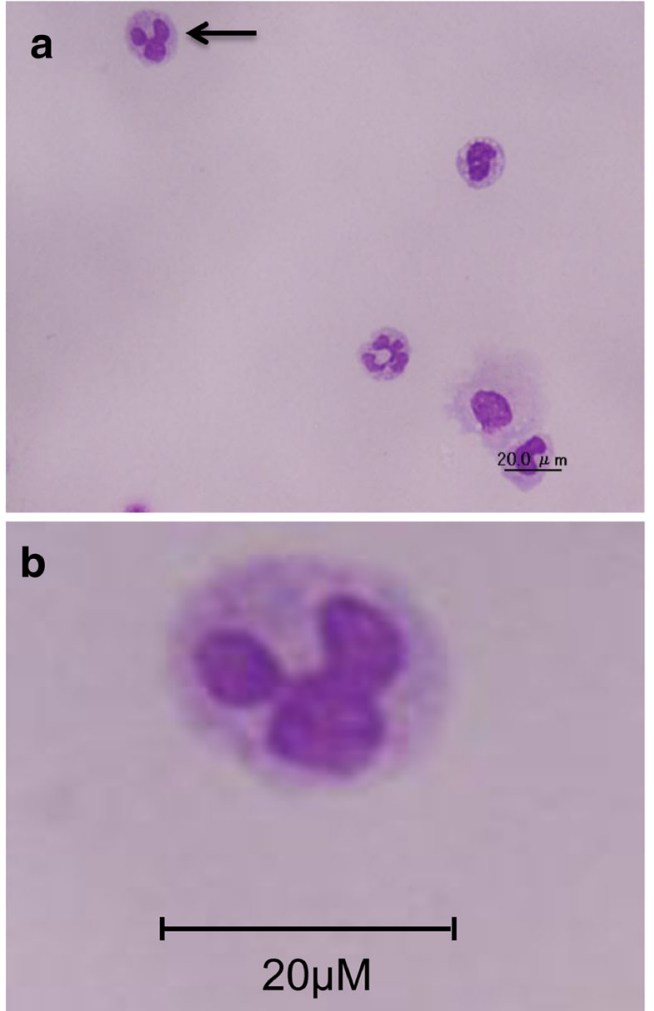

Fig. 1 Unusual neutrophil segmentation (arrow) in a stained smear (May Grunwald/Giemsa) taken from a bone marrow cytospin sample

Conflict of interest The authors declare no conflicts of interest.

\section{Reference}

Bitar C, Saba N (2014) Neutrophil numerals. Blood 123:1635

Mice were housed and handled under conditions stipulated by the Institutional Animal Care and Use Committee (IACUC) of Kyushu University, Japan. 